\title{
Electronic Properties of Graphene/hBN Heterostructures with in-Plane Displacement
}

\author{
A.P. Durajski* And A.E. Auguścik \\ Institute of Physics, Czȩstochowa University of Technology, \\ al. Armii Krajowej 19, 42-200 Czȩstochowa, Poland \\ Doi: $10.12693 /$ APhysPolA.138.136 \\ *e-mail: adurajski@wip.pcz.pl
}

\begin{abstract}
Graphene has very good electronic properties, however, it is challenging to make it into nanoelectronic devices. A substrate can be introduced to solve this problem, whereby, this can open up the electronic bandgap, thereby increasing the usability of graphene for numerous potential applications in novel devices. In this paper, we perform first-principles calculations for heterostructures composed of monolayer graphene and hexagonal boron nitride $(\mathrm{hBN})$. We examine the influence of mutual in-plane displacement of graphene and $\mathrm{hBN}$ layers on the electronic properties of the whole system. The horizontal shift by a multiple of half the carbon-carbon bond length indicates that configuration with one $\mathrm{C}$ atom situated over the $\mathrm{B}$ atom and the other $\mathrm{C}$ atom over the hollow site of $\mathrm{hBN}$ is energetically most favorable. Moreover, we observed explicitly the bandgap opening in graphene/hBN systems to roughly $64 \mathrm{meV}$ for the configuration with one $\mathrm{C}$ atom above $\mathrm{B}$ atom and the other $\mathrm{C}$ atom above $\mathrm{N}$ atom which provides theoretical direction for further experimental verification.
\end{abstract}

topics: graphene/hBN heterostructure, electronic properties, DFT calculations

\section{Introduction}

In recent years, two-dimensional (2D) materials such as graphene, hexagonal boron nitride, silicene, germanene, phosphorene, transition metal dichalcogenides and so on, have attracted the widespread interest of researchers as they exhibit dramatically distinctly different physical properties from their bulk counterparts [1]. Among these structures, due to the easy process of isolation by the exfoliation of graphite, the graphene became one of the most popular 2D material [2]. The superior optical, electronic and thermal properties of this 2D layer of carbon atoms arranged in a hexagonal lattice, have paved the way for practical applications in nanoelectronics, electrochemistry, sensors, catalysts, transistors, and supercapacitors [3].

However, such properties have not been fully exploited in many potential applications. This is partly due to the fact that graphene being a gapless semiconductor is not always suitable for use in its pristine form. Therefore, it is essential to generate a finite gap in the energy dispersion at the Dirac point [4] and precise control over it. A number of methods have been proposed in the literature to modify the graphene and its electronic structure [5-7]. One of the promising approaches that allows open up the bandgap is the chemical doping (adsorption of foreign atoms to the graphene surface or substitutional doping through foreign atoms into the carbon lattice of graphene). In fact, chemically modified graphene exhibits quite interesting properties, such as superconductivity, ferromagnetism, and enhanced optical features, which promote a widespread application of graphene-based materials in different technological areas $[8,9]$. Another way to modulate the electronic structure of graphene is the formation of heterostructures with other two-dimensional materials.

In the past few years, hexagonal boron nitride $(\mathrm{hBN})$ has emerged as an excellent substrate for graphene because monolayer graphene and hBN have a similar lattice structure, with a lattice mismatch less than $2 \%[10,11]$. While graphene is a semimetal with a zero bandgap, $\mathrm{hBN}$ is an insulator with a large energy gap dispersed in the range between 3.6 and $7.1 \mathrm{eV}[12]$. The ab initio density functional calculations performed by Giovannetti et al. and Fan et al. show that the most stable configuration has one carbon atom above a boron atom, and the other centered above an hBN ring $[10,13]$. Moreover, it was found that the energy gap of graphene is tunable from 0 to $0.55 \mathrm{eV}$ and sensitive to the stacking order and interlayer distances of the graphene/hBN bilayer $[13,14]$.

In this study, we investigated of the energetic and electronic structures of graphene/hBN heterostructure. We explore the possibility of tuning the electronic properties by mutual out-of-plane and in-plane displacement of graphene and hBN layers. The energy minimization shows that the distance between graphene and hBN layers is strongly influenced by the mutual in-plane positions of 
monolayers. This ultimately leads to achieving the energy gap of $64 \mathrm{meV}$ for the configuration with one $\mathrm{C}$ atom above $\mathrm{B}$ atom and the other above $\mathrm{N}$ atom.

The paper is organized in the following way. In Sect. 2 we present the details of computational methods. In Sect. 3 we study the effect of mutual in-plane displacement of graphene and hBN layers on the energy gap. Finally, the paper is summarized in Sect. 4.

\section{Computational methods}

The first-principles calculations were performed using the generalized gradient approximation (GGA) of the Perdew-Burke-Ernzerhof (PBE) functional [15], as it was implemented in the QUANTUM ESPRESSO package $[16,17]$. The projector-augmented wave (PAW) pseudopotentials [18] were adopted for electron-ion interactions. The kinetic energy cutoff of 60 Ry was applied for the plane-wave expansion. For structural relaxation and electronic structure calculation, the Brillouin zone was sampled with a $36 \times 36 \times 1$ Monkhorst-Pack $k$-point mesh. The initial atomic structure configuration with one $\mathrm{C}$ atom over the $\mathrm{B}$ atom and the other $\mathrm{C}$ atom over the hollow site of $\mathrm{hBN}$ was fully relaxed until reaching the forces less than $0.001 \mathrm{eV} / \AA$. A vacuum space of at least $17 \AA$ was included in the out-of-plane direction to eliminate the interaction between spurious images of the graphene/hBN structures.

\section{Results and discussion}

Even though graphene and hBN are isostructural, in several experimental preparations of graphene on $\mathrm{hBN}$ substrate, the lattice constant of $\mathrm{hBN}$ is about $1.8 \%$ larger than that of graphene (C-C and $\mathrm{B}-\mathrm{N}$ bond lengths are of $1.420 \AA$ and $1.446 \AA$, respectively) [11]. In monolayer systems, which are willing to accommodate, the lattice mismatch between graphene and hBN is resolved by isotropically averaging of the graphene and $\mathrm{hBN}$ bond lengths. The fully relaxed lattice constant of graphene $/ \mathrm{hBN}$ structure is $1.425 \AA$ for the energetically most favorable configuration where one carbon is on top of the boron atom and one at the center of BN hexagon (see configuration I in Fig. 1). Of course, different crystallographic stacking orientations are also possible. In Fig. [1] we present other configurations created by the mutual in-plane displacement of graphene and hBN layers through the horizontal shift of hBN by a multiple of half bond length (0.7127 ̊).

The different stacking manner leads to different interlayer distances between graphene and hBN when minimizing the total energy of the individual geometries [19]. In Fig. 2 we show the total energy as a function of the distance between graphene and $\mathrm{hBN}$, for all investigated stacking configurations. We find that in the whole range of distances

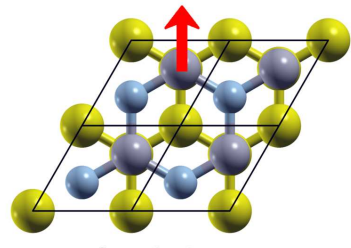

configuration I

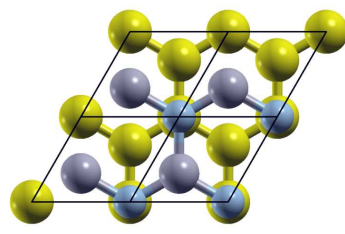

configuration III

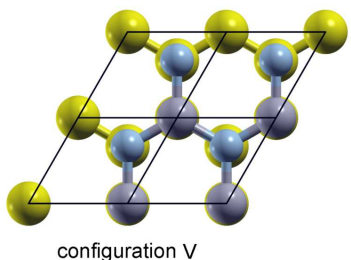

configuration $\mathrm{V}$
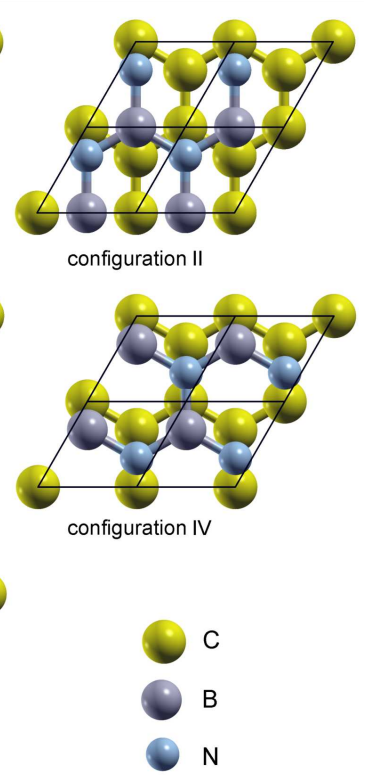

Fig. 1. Stacking configurations of graphene $/ \mathrm{hBN}$ heterostructures. The red arrow indicates the direction of the shift of the $\mathrm{hBN}$ layer in relation to the graphene sheet.

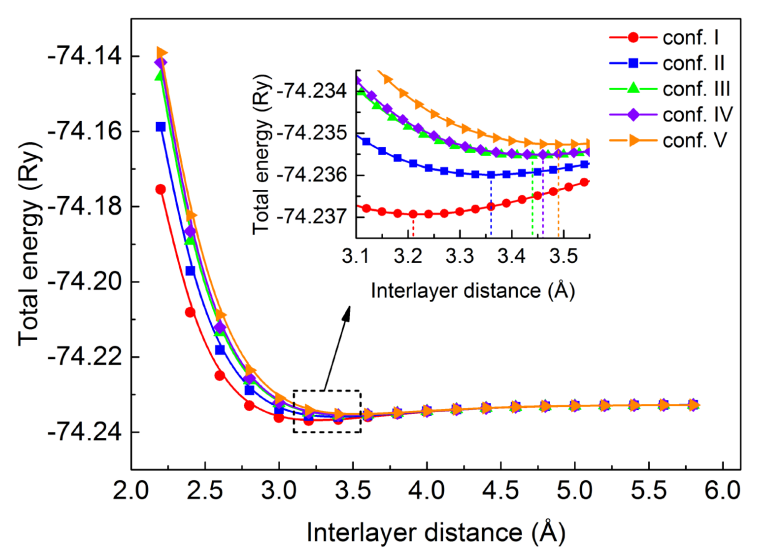

Fig. 2. Total energy as a function of the interlayer distance between graphene and hBN with different stacking manners.

the total energy is the lowest for the configuration I. The equilibrium distances of all configurations are collected in Table I. It should be noted that the obtained results for stacking configurations I, III and V (3.21 $\AA, 3.44 \AA$ and $3.49 \AA$, respectively) are consistent with previous calculations (3.22 $\AA$, $3.40 \AA$ and $3.50 \AA$, respectively) conducted by Giovannetti et al. using Vienna ab initio simulation package [10].

To study the effect of mutual in-plane displacement of graphene and hBN layers on the energy gap in this system, we calculate the band dispersion of different stacking graphene/hBN configurations with lowest energy distances between monolayers. 
TABLE I

Comparison of the interlayer distance $(\mathrm{d})$, total energy difference $\left(\Delta \mathrm{E}=\mathrm{E}-\mathrm{E}_{\mathrm{conf} . \mathrm{I}}\right)$, and energy gap $\left(E_{g}\right)$ for studied stacking configurations of graphene $/ \mathrm{hBN}$ heterostructure.

\begin{tabular}{c|c|c|c|c}
\hline \hline Conf. & $\begin{array}{c}\text { Displacement } \\
{[\AA]}\end{array}$ & $\begin{array}{c}d \\
{[\AA]}\end{array}$ & $\begin{array}{c}\Delta E \\
{[\mathrm{meV}]}\end{array}$ & $\begin{array}{c}E_{g} \\
{[\mathrm{meV}]}\end{array}$ \\
\hline I & 0 & 3.21 & 0 & 59.0 \\
II & 0.7127 & 3.36 & 12.7893 & 36.5 \\
III & 1.4254 & 3.44 & 19.0479 & 45.2 \\
IV & 2.1381 & 3.46 & 19.3200 & 58.8 \\
V & 2.8508 & 3.49 & 22.5854 & 64.0
\end{tabular}

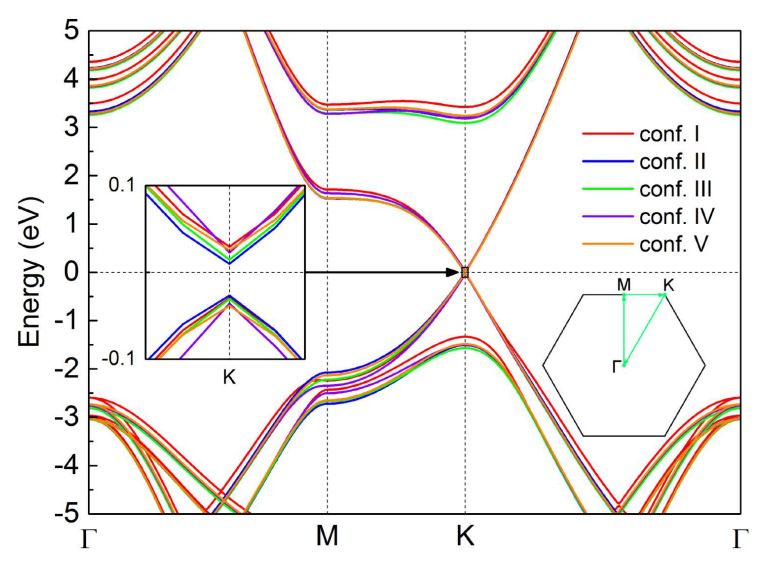

Fig. 3. Calculated electronic band structures of five graphene/hBN configurations. The Fermi level is set at $0 \mathrm{eV}$. The insets show a zoom around $\mathrm{K}$ point and the associated Brillouin zone.

The calculated electronic band structures along the high symmetry $\Gamma-\mathrm{M}-\mathrm{K}-\Gamma$ direction are shown in Fig. 3 and the obtained energy gaps are collected in Table I. We found that the direct bandgap of the graphene/h-BN heterostructure could be strongly modulated through the mutual in-plane displacement of monolayers.

It is know from literature that decreasing the distance between the graphene sheet and the h-BN surface increases the gap $[10,13,14]$. In our study, we found that the energy gap can be modulated not only by changing the distance between graphene and hBN layers but also through the mutual inplane displacement of monolayers. As we can see, in Table the largest energy gap of $64 \mathrm{meV}$ is found for the configuration IV with one carbon atom above a boron atom and the other above a nitrogen atom.

\section{Conclusions}

Using first-principles calculations, we show that the bandgap of graphene/hBN structure can be modulated effectively by tuning the stacking arrangement. Starting from the initial atomic structure configuration with one $\mathrm{C}$ atom over the $\mathrm{B}$ atom and the other $\mathrm{C}$ atom over the hollow site of $\mathrm{hBN}$ the configurations II-V were achieved by step translating $\mathrm{hBN}$ monolayer for a distance of multiples of $1 / 2$ graphene lattice constant with respect to graphene along $\mathrm{C}-\mathrm{C}$ bond orientation. The procedure of energy minimization shows that the distance between graphene and hBN layers is strongly influenced by the mutual in-plane positions of monolayers. This ultimately leads to achieving the energy gap of $64 \mathrm{meV}$ for the configuration with one carbon atom above a boron atom and the other above a nitrogen atom. We expect that our studies will enhance the understanding of graphene/hBN heterostructure materials and promote the experimental verification of presented herein results.

\section{Acknowledgments}

A.P. Durajski acknowledges the financial support from the Czestochowa University of Technology under Grant No. BS/MN-200-301/2019 and from the Polish Ministry of Science and Higher Education under the scholarship for young outstanding scientists No. 406/STYP $/ 13 / 2018$. This research was supported in part by PLGrid Infrastructure.

\section{References}

[1] R. Mas-Ballesté, C. Gómez-Navarro, J. Gómez-Herrero, F. Zamora, Nanoscale 3, 20 (2011).

[2] A. Geim, K. Novoselov, Nature Mater. 6 , 183 (2007).

[3] X.J. Lee, B.Y.Z. Hiew, K.C. Lai, L.Y. Lee, S. Gan, S. Thangalazhy-Gopakumar, S. Rigby, J. Taiwan. Inst. Chem. Eng. 98, 163 (2019).

[4] S. Sahu, G.C. Rout, Int. Nano. Lett. 7, 81 (2017).

[5] S. Ullah, A. Hussain, W. Syed, M.A. Saqlain, I. Ahmad, O. Leenaerts, A. Karim, RSC Adv. 5, 55762 (2015).

[6] N. Ullah, R.Q. Zhang, G. Murtaza, A. Yar, A. Mahmood, Solid State Commun. 246, 54 (2016).

[7] J.-S. Park, H.J. Choi, Phys. Rev. B 92, 045402 (2015).

[8] S. Agnoli, M. Favaro, J. Mater. Chem. A 4, 5002 (2016).

[9] M. Goudarzi, S.S. Parhizgar, J. Beheshtian, Opto-Electron. Rev. 27, 130 (2019).

[10] G. Giovannetti, P.A. Khomyakov, G. Brocks, P.J. Kelly, J. van den Brink, Phys. Rev. B 76, 073103 (2007).

[11] A. Pakdel, C. Zhi, Y. Bando, D. Golberg, Mater. Today 15, 256 (2012). 
[12] V.L. Solozhenko, A.G. Lazarenko, J.-P. Petitet, A.V. Kanaev, J. Phys. Chem. Solids 62, 1331 (2001).

[13] Y. Fan, M. Zhao, Z. Wang, X. Zhang, H. Zhang, Appl. Phys. Lett. 98, 083103 (2011).

[14] E. Kan, H. Ren, F. Wu, Z. Li, R. Lu, C. Xiao, K. Deng, J. Yang, J. Phys. Chem. C 116, 3142 (2012).
[15] J.P. Perdew, K. Burke, M. Ernzerhof, Phys. Rev. Lett. 77, 3865 (1996).

[18] P.E. Blochl, Phys. Rev. B, 50, 17953 (1994).

[19] K. Zollner, M. Gmitra, J. Fabian, Phys. Rev. B 99, 125151 (2019). 